\title{
Capacitismo, covid-19 y dignidad: derecho a la salud e inclusión en tiempos de pandemia ${ }^{1}$
}

\section{Manuel Aparicio Payá}

Facultad de Filosofía, Universidad de Murcia

manuel.aparicio@um.es

\section{Mario Toboso Martín}

Instituto de Filosofía, Centro de Ciencias Humanas y Sociales, CSIC

mario.toboso@csic.es

Covid-19k eragindako pandemiak eragindako osasun publikoko krisia giza eskubideen krisi bihurtu da, eta bidegabeki eragin die dibertsitate funtzionala duten pertsonei eta adineko pertsonei. Lan honetan uste dugu azken krisi honen azpian aurreiritzi kapazitistaren eta aurreiritzi adinaren arteko elkargunea aurkitzen dugula. Lehenengo zatian, kapazitismoa definitu ondoren, gizarte-talde horien osasun-laguntzaren ex ante bazterketaren kritika arauemailea egiten dugu. Duintasunaren ideiaren azpian dagoen berdintasunezko unibertsaltasunaren ikuspuntua aldezten dugu, giza eskubideen oinarria eta horietan oinarritutako bioetika. Azkenik, pandemiaren aurrean jarrera inklusiboa lantzen dugu, bai osasun-praktikaren justiziaren ikuspegitik, bai gizarte-justiziaren ikuspuntu zabalenetik.

\section{GAKO-HITZAK:}

Kapazitismoa, pandemia, aniztasun funtzionala duten pertsonak, duintasuna, giza eskubideak, osasunerako eskubidea.
La crisis de salud pública provocada por la pandemia causada por la covid-19 se ha convertido en una crisis de derechos humanos que ha afectado injustamente a personas con diversidad funcional y a personas ancianas. En este trabajo consideramos que en el subsuelo de esta última crisis encontramos la intersección entre el prejuicio capacitista y el prejuicio edadista. En una primera parte, después de definir el capacitismo, realizamos una crítica normativa de la exclusión ex ante de la asistencia sanitaria de estos grupos sociales. Defendemos el punto de vista del universalismo igualitario subyacente en la idea de dignidad, fundamento de los derechos humanos y de una bioética centrada en ellos. Finalmente, abordamos el proceder inclusivo frente a la pandemia, tanto desde el punto de vista de la justicia de la práctica sanitaria como desde el punto de vista, más amplio, de la justicia social.

\section{Palabras Clave:}

Capacitismo, pandemia, personas con diversidad funcional, dignidad, derechos humanos, derecho a la salud

\footnotetext{
${ }^{1}$ Este trabajo se enmarca dentro del proyecto de investigación "Capacitismo" (FFl2017-88787-R), financiado por el Ministerio de Ciencia e Innovación del Gobierno de España.
} 


\section{Introducción}

La crisis sanitaria que está azotando actualmente al mundo, producida por la explosiva expansión del coronavirus SARS-CoV-2, ha derivado en una crisis humana y social de ámbito global (ONU, 2020a). Una ramificación de esta que ha emergido con crudeza ha sido el trato discriminatorio sufrido por las personas con diversidad funcional en las respuestas dadas para hacerles frente (ONU, 2020c). A pesar de la protección jurídica vinculante que tiene este grupo social, en virtud de la vigencia de la Declaración Universal de los Derechos Humanos (DUDH) de 1948 y de la Convención sobre los Derechos de las Personas con Discapacidad (CDPD), aprobada por la ONU en 2006, diversas organizaciones han denunciado el trato injusto hacia tales personas en relación al conjunto de sus derechos, en particular al igual derecho a la protección de su salud². En ese sentido, la Alta Comisionada de la ONU para los Derechos Humanos, Michelle Bachelet, mostró su inquietud por "determinados informes que indican que durante esta pandemia las vidas de las personas con discapacidad han sido menos valoradas que las de otros pacientes" (ONU, 2020d). En nuestro ámbito geográfico, el Parlamento Europeo, reconoció que "durante la crisis de la covid-19, las personas con discapacidad han experimentado graves problemas y violaciones de derechos, como perturbaciones en los servicios de asistencia, atención y apoyo personales, un acceso desigual o nulo a la información relativa a la salud y a la atención sanitaria" (Parlamento Europeo, 2020: consideración Y).

También la voz de las personas con diversidad funcional se ha alzado para mostrar la enorme desproporción con que la pandemia las ha golpeado. En una opinión coincidente, esta voz revela no solo una catástrofe sanitaria, sino también una "catástrofe de derechos humanos" en España y a nivel internacional (CERMI, 20203). Todas estas manifestaciones ponen de relieve la grave problemática moral y jurídico-política que enfrenta el mundo provocada por la catástrofe sanitaria y sus consecuencias, que acentúa la realidad cotidiana vivida por buena parte de las personas con diversidad funcional. Lo que ahora sale a la

${ }^{2}$ La relatora especial sobre los derechos de las personas con discapacidad, Catalina Devandas, es igualmente clara al respecto, al afirmar que "las personas con discapacidad tienen las mismas necesidades de salud que las demás personas, entre otras, la promoción de la salud, la atención preventiva, el diagnóstico, el tratamiento y la rehabilitación. También pueden tener otras necesidades de salud ocasionadas por sus deficiencias y otros determinantes subyacentes de la salud, como la pobreza, la discriminación, la violencia y la exclusión social” (ONU, 2018: párr. 4). En lo que sigue se destaca el agravamiento de enfermedades debido a un entorno social inadecuado, circunstancia que estimamos relevante en el contexto de la crisis sanitaria de la covid-19: "Si bien algunas deficiencias evolucionan de forma inevitable con el tiempo, un entorno físico y social inadecuado puede agravar las enfermedades primarias o exacerbar las consecuencias secundarias de dichas enfermedades".

${ }^{3}$ CERMI es voz representativa de este grupo social y tiene un papel en la reivindicación ético-política de los derechos humanos, al ser reconocida en 2011 por el Estado español como mecanismo independiente y de seguimiento de la aplicación de la CDPD. superficie con más intensidad y es visibilizado a través de numerosos informes institucionales y denuncias de medios de comunicación, es el trato desigual y excluyente dado en numerosas ocasiones a dichas personas, a pesar de los avances sociales de las últimas décadas. La injusticia moral y jurídicopolítica, que se ha manifestado ahora palmariamente en forma de una más restringida inclusión social y una grave discriminación en el ejercicio igualitario del derecho a la salud, es una de las caras de las crisis que están haciendo resquebrajarse las bases sobre las que se erigen las sociedades (ONU, 2020a). Desde la perspectiva de lo correcto, esta situación exige reparación y cambios institucionales hacia el futuro. En este trabajo sostenemos que dicha problemática puede conceptuarse relacionándola con un viejo prejuicio naturalizado, a través de raíces histórico-culturales, en las estructuras sociales ${ }^{4}$ : el prejuicio capacitista. La dimensión natural de la covid-19 parece concordar con un naturalizado capacitismo instalado en el imaginario social, ignorando o eludiendo la responsabilidad de las estructuras sociales y de las iniciativas personales en ponerles freno $0^{5}$. La valoración cultural desigual de las diferentes capacidades que tienen los seres humanos, en claro detrimento de los considerados "menos capaces", viene a coincidir con la restricción a su igualitaria inclusión social y con la discriminación en su derecho a la salud en la negación del valor fundamental de la igual dignidad humana ${ }^{6}$. Este valor, puesto de relieve en la DUDH y en la CDPD, en su doble cara de valor moral y valor jurídico-político (Habermas, 2012), exige el cuestionamiento del capacitismo.

4 "La exclusión [...] puede estar insertada en la cultura y las estructuras sociales dominantes, que la 'naturalizan'” (Etxeberria, 2018: 282).

5 La Oficina del Alto Comisionado de las Naciones Unidas para los Derechos Humanos, en el informe acerca de la toma de conciencia impulsada en el artículo 8 de la CDPD (ONU, 2019b: párr. 15), señala que, al igual que sucede con el racismo, el sexismo y el edadismo, el "capacitismo" se conforma mediante un sistema de creencias que origina las actitudes y estereotipos negativos, así como el estigma, que devalúan a las personas con discapacidad basándose en sus deficiencias. Es clave comprender que para el capacitismo "las personas con discapacidad merecen menos respeto y consideración, son menos capaces de contribuir y participar, y tienen un valor inherente inferior al de las demás personas. Arraigado en las instituciones, los sistemas y la cultura general de la sociedad, coarta las posibilidades de las personas con discapacidad y menoscaba su inclusión en la vida de la comunidad".

${ }^{6}$ En el informe sobre el derecho a la salud, la relatora especial, Catalina Devandas, denuncia que en muchos países se discrimina a las personas con discapacidad en el acceso a tratamientos médicos, por motivo de su discapacidad. Aunque las justificaciones dadas para ello van desde la falta de accesibilidad hasta una cobertura insuficiente de seguro médico, se trata de prácticas discriminatorias basadas en la premisa de que las personas con discapacidad tienen menos valor que las demás personas o disfrutan de una calidad de vida menor. "A muchos niños y adultos con discapacidad no se les ofrece o se les niega el tratamiento, incluso cuando se trata de tratamientos que podrían salvar o prolongar la vida, ya que se considera que son intervenciones sin sentido e ineficaces" (ONU, 2018: párr. 38). El estudio de Gerhart et al. (1994), mencionado por la relatora especial, reveló que muchos proveedores sanitarios no apoyan intervenciones estándar de emergencia, como toracotomías, en pacientes con lesiones recientes de la médula espinal. "No garantizar el acceso a tratamiento y medicación vitales para aliviar el dolor y el sufrimiento representa una amenaza para los derechos a la vida, a la salud y a no ser sometido a tratos crueles, inhumanos y degradantes". 
En este artículo nos centramos, en primer lugar, en un análisis conceptual del prejuicio capacitista, señalando la repercusión que tiene en las personas con discapacidad. En segundo lugar, realizamos un análisis general de la exclusión de las personas con diversidad funcional en la respuesta a la pandemia, considerando un doble marco teórico: el enfoque ético-político de Derechos Humanos y el ámbito de la reflexión bioética. Apoyándonos en el planteamiento universalista igualitario que subyace en el pensamiento jurídico-político, de base normativa, de Habermas, defendemos una perspectiva inclusiva en la respuesta a la pandemia, fundada en la idea moral-jurídica de dignidad, común a ambos marcos teóricos. La inclusión igualitaria de todos los ciudadanos, fundada en dicha normatividad moral, constituye la base legítima de una comunidad política erigida jurídicamente sobre los derechos humanos. La pertinencia de un universalismo igualitario abierto a la diferencia (Habermas, 2008b) se sostiene sobre la denuncia de que la crisis sanitaria constituye también una crisis de derechos humanos. Por tanto, una crisis jurídico-política del sistema democrático. A su vez, el valor de la dignidad humana y el derecho a la salud derivado de ella juegan un papel relevante en los debates bioéticos sobre la problemática del tratamiento médico de los pacientes con coronavirus. Recurriendo a ambos marcos teóricos, sostenemos que la idea de dignidad proporciona una potente base para la crítica normativa que cuestiona, por injusto, el trato discriminatorio hacia las personas con diversidad funcional y las personas ancianas ocurrido durante la actual pandemia.

En tercer lugar, exploramos el debate sobre los criterios de triaje médico en la situación de catástrofe sanitaria, cuestionando desde consideraciones epistémicas y normativas el capacitismo subyacente en algunos planteamientos. Justificamos que el valor de igual dignidad en la diferencia, incluido en la CDPD, debe seguir vigente en la atención sanitaria durante la pandemia. También exploramos la cuestión de los determinantes sociales de salud (DSS) puestos de relieve desde la epidemiología social, en la medida en que han de ser tenidos en cuenta en la corrección de desigualdades que puedan incidir en futuras pandemias.

\section{El prejuicio capacitista}

El capacitismo ("ableism”) denota un discurso social y cultural dominado por una actitud que devalúa la discapacidad, frente a la valoración de la integridad corporal, que es equiparada a una supuesta condición esencial humana de normalidad. Puede definirse como un conjunto de creencias, procesos, representaciones y prácticas basado en el favoritismo y el privilegio de ciertas capacidades, que produce una interpretación particular de uno mismo, del propio cuerpo, de la relación con los demás y con el medio ambiente (Wolbring, 2008b: 252). La clase particular de sujeto y de cuerpo que se proyecta normativamente como lo deseable y típico de la especie y, por lo tanto, como lo que es esencial y plenamente humano, produce que la discapacidad sea considerada como una condición devaluada y devaluante del ser humano (Campbell, 2001: 44). El capacitismo se basa, pues, en el prejuicio de que las personas con discapacidad son inferiores a las personas sin discapacidad (Linton, 1998: 9), se relaciona con la categorización médica de las personas con discapacidad como deficientes y minusválidas, y conduce al objetivo de rehabilitarlas 0 , incluso, de prevenir su nacimiento (Wolbring, 2008b), al asumir la creencia de que la discapacidad, de cualquier tipo que sea, es inherentemente rechazable (Campbell, 2008).

En la mayoría de las ocasiones, el capacitismo es un discurso implícito pero que actúa de manera muy efectiva, puesto que impregna gran parte del pensamiento y de los ámbitos de la sociedad, entre ellos, de especial importancia, el actitudinal, generando barreras que son señaladas claramente en el apartado "e" del preámbulo de la CDPD:

"Reconociendo que la discapacidad es un concepto que evoluciona y que resulta de la interacción entre las personas con deficiencias y las barreras debidas a la actitud y al entorno que evitan su participación plena y efectiva en la sociedad, en igualdad de condiciones con las demás".

El capacitismo favorece actitudes sociales negativas hacia las personas con discapacidad, que son objeto de bajas expectativas, de estigmatización, de estereotipos y prejuicios. Ello conduce a la discriminación, al uso de un lenguaje peyorativo y a prácticas nocivas y violentas. La CDPD incluye en su artículo 8 una disposición transversal sobre la toma de conciencia en relación con las habilidades, las aportaciones a la sociedad y los derechos de las personas con discapacidad. Esta toma de conciencia es fundamental para superar la concepción desfasada de la discapacidad todavía presente en la legislación y en la práctica, como en los modelos médico y caritativo (ONU, 2019b: párr. 72-73).

En tanto discurso social y cultural, el capacitismo tiene raíces en teorías y enfoques filosóficos, sociológicos, éticos, antropológicos, jurídicos, científicos y de otro tipo, en relación con nociones como la autonomía personal, la capacidad, la salud, la individualidad, la subjetividad, la agencia, etc. Estas nociones, que están del lado de los privilegios, correlacionan con otras que caen del lado de la discriminación, como son la minusvalía, la dependencia, la discapacidad, la enfermedad, la incapacidad y otras, que se han venido planteando a lo largo del tiempo, en paralelo con el desarrollo de las teorías y enfoques correspondientes.

Atendiendo a estándares normativos y estrictos de apariencia, funcionamiento y comportamiento, el pensamiento capacitista considera desgraciada la experiencia de la discapacidad como una tragedia que conlleva sufrimientos y desventajas y que, de forma inapelable, resta valor a la vida humana. Como 
consecuencia de ello, se establece la creencia de que la calidad de vida de las personas con discapacidad es ínfima, que esas personas no tienen ningún futuro y que nunca se sentirán realizadas y ni felices (ONU, 2019c: párr. 9).

En los últimos cincuenta años, el movimiento de defensa de los derechos de las personas con discapacidad ha tratado de luchar contra estas percepciones negativas profundamente arraigadas, señalando la raíz del problema en el hecho de que la sociedad no elimine las barreras, que no preste el apoyo necesario y no se acepte la discapacidad como parte de la diversidad humana, de acuerdo con el artículo 3, que enuncia los principios generales de la CDPD. Las narraciones de las personas con discapacidad se consideran subjetivas e inadecuadas para orientar la adopción de decisiones objetivas, y el desequilibrio de poder subyacente invalida su experiencia real de vida. Se hacen oídos sordos a sus reivindicaciones para que se reconozcan sus derechos, y no se les proporcionan espacios de comunicación ni de debate para que se tengan en cuenta o para poner en tela de juicio el capacitismo (ONU, 2019c: párr. 15).

La idea de capacitismo evolucionó a partir de los movimientos por los derechos civiles de las personas con discapacidad en los Estados Unidos y el Reino Unido, durante las décadas de 1960 y 1970 (Wolbring y Guzmán, 2010). Impulsado como un concepto de uso análogo a los de sexismo y racismo, pretendía evidenciar y combatir los prejuicios y la discriminación hacia las personas con discapacidad, cuyos cuerpos y capacidades se señalaban como “deficientes". Tanto el discurso acerca de los derechos de las personas con discapacidad como los estudios sobre la discapacidad en el ámbito académico cuestionaron el favoritismo hacia las capacidades de un supuesto cuerpo normativo, base del capacitismo, que considera esencialmente valiosas ciertas capacidades que se deben poseer, conservar o adquirir.

El favoritismo y privilegio hacia unas capacidades esenciales, por encima de otras, se ha utilizado a lo largo del tiempo para justificar también la discriminación hacia grupos sociales distintos de las personas con discapacidad, que exhiben diferencias reales o percibidas de tales capacidades, y ha conducido a la justificación de otros "ismos", como el racismo o el sexismo (Wolbring, 2008a). Si bien el racismo y el sexismo pueden carecer de legitimidad social en el mundo actual supuestamente “civilizado", los preceptos que regulan la civilidad moderna continúan, por el contrario, permitiendo una orientación marcadamente capacitista (Cherney, 2011).

El capacitismo es uno de los “ismos” más arraigados y aceptados socialmente (Wolbring y Guzmán, 2010). Los juicios de valor sobre las capacidades están tan presentes en la sociedad que sus efectos excluyentes apenas son percibidos ni cuestionados.
Incluso quienes son marginados por el capacitismo, caen, paradójicamente, en el mismo discurso para defenderse o exigir cambios en su situación: "nosotros somos tan capaces como vosotros [...] con los apoyos necesarios, podemos ser tan capaces como cualquiera" (Wolbring, 2008b).

El capacitismo impregna el pensamiento actual y opera como un discurso de poder y de dominación transmitido a través de dispositivos retóricos como el lenguaje, las imágenes y todos los sistemas de representación. Está tan presente que llega a parecer natural, inevitable y, en última instancia, moralmente aceptable y necesario para el funcionamiento normal de la sociedad (Cherney, 2011). Vivimos en un mundo capacitista que asume la ausencia de discapacidad como el estado "natural" del ser humano y considera, además, este estado como altamente deseable, acompañado de todo tipo de privilegios e institucionalmente recompensado (McRuer, 2016: 140).

A pesar de su amplísima presencia, el capacitismo, en tanto discurso social y cultural, permanece oculto, en una especie de omnipresente invisibilidad. La opinión pública cuestiona cada vez más los discursos discriminatorios, pero el capacitismo sigue legitimando la retórica que da lugar a distintas formas de discriminación (ONU, 2019b: párr. 15). Las posturas capacitistas dominan importantes debates que afectan a los derechos de las personas con discapacidad. Ideas normativas y desfasadas sobre la normalidad se siguen imponiendo en deliberaciones médicas, jurídicas y filosóficas, incluyendo las que conciernen a prácticas científicas y médicas tan delicadas como el cribado prenatal, la edición génica, la permisividad de intervenciones invasivas, dolorosas o irreversibles, la denegación o retirada de intervenciones terapéuticas de soporte vital y la muerte asistida. Con frecuencia, tales deliberaciones tienen lugar principalmente en el ámbito de la bioética (ONU, 2019c: párr. 16).

\section{Derechos humanos y bioética: crítica normativa de la discriminación sanitaria en la crisis pandémica}

La experiencia de injusticia sufrida por el grupo social de personas con diversidad funcional y por personas ancianas ${ }^{7}$ en el contexto de la crisis pandémica muestra la distancia existente entre las bases normativas igualitarias proclamadas por

7 El $46 \%$ de las personas con 60 años o más tiene algún tipo de impedimento (ONU, 2020b). Por otra parte, como ejemplo de microdatos de la confluencia entre impedimento y edad, un estudio (Suárez, Matías y Comas, 2020) estima, en ausencia de datos desagregados completos, que entre el $61 \%$ y el $76 \%$ de personas que viven en residencias de ancianos en España tienen demencia, considerando que existe evidencia científica emergente de una mayor vulnerabilidad a la covid-19 en dichas personas. Tampoco existen datos desagregados completos por parte del movimiento asociativo (CERMI, 2020) en relación con personas con diversidad funcional en residencias, en sus casas o en hospitales (CERMI, 2020). 
la sociedad y la realidad efectivamente vivida. No obstante, las organizaciones que han denunciado el desajuste no renuncian a seguir ahondando en dicha normatividad, entendiendo que la corrección de tal falla únicamente será posible a partir de la orientación que aquella proporciona. Las argumentaciones prácticas, éticas y jurídicas, reflejadas en los diferentes informes sobre la repercusión de la covid-19 en dicho grupo social provienen fundamentalmente de dos fuentes: por un lado, la puesta de relieve de los derechos humanos que les asisten, modulados en la CDPD; por otro, la reflexión procedente del ámbito de la bioética. El terreno ético común que entrelaza ambos marcos teóricos permite encontrar en el valor de la dignidad un doble aspecto de crítica normativa, ya que, desde una perspectiva kantiana, el respeto a la igual dignidad de la persona constituye el fin limitativo de las acciones que la menoscaben, así como el fin positivo que debe ser perseguido para protegerla (Cortina, 2009a). Se abre, pues, una doble perspectiva de crítica normativa: por un lado, el menoscabo de la dignidad que supone la denegación del derecho a la salud; por otro, el menoscabo de la dignidad producido al no haberse creado iguales condiciones de acceso a la salud.

\subsection{Crítica de la discriminación desde los derechos humanos}

Hay que tener en cuenta que los derechos humanos, formulados históricamente con pretensión de universalidad a partir de la DUDH de 1948, se reafirman en dicha universalidad al extenderse a este marginado grupo social en la CDPD. El discurso axiológico-normativo subyacente en este documento asume definitivamente una nueva concepción de la discapacidad: sin negar la experiencia del impedimento, concibe a las personas con alguno de sus tipos como sujetos de iguales derechos, señalando la responsabilidad de la sociedad en su inclusión, a través de una clara hoja de ruta para las políticas públicas (Palacios, 2008; Degener, 2016). Lo que ha hecho aguas ahora, y es necesario taponar para avanzar en una respuesta inclusiva frente a la covid-19 (ONU, 2020b), es el traslado al imaginario social de esta nueva concepción:

Las sociedades avanzadas, occidentales y con democracias consolidadas, como la española, siguen viendo a las personas con discapacidad desde la óptica de la prescindencia o desde el modelo médico rehabilitador y poco o nada ha calado el enfoque de derechos, basado en la dignidad y la autodeterminación de las propias personas que propugna la Convención. (CERMI, 2020)

\footnotetext{
${ }^{8}$ La CDPD no implica el establecimiento de nuevos derechos humanos; más bien constituye una modulación de estos a las diversas características fácticas diferenciales que presentan las personas con diversidad funcional (Palacios, 2008; Degener, 2016).
}

Los derechos humanos no se agotan en su dimensión jurídica al ser incorporados al ámbito legislativo interno de un Estado. Sin detrimento de que, por su traducción jurídica, pueda apelarse a ellos para denunciar posibles injusticias tomando como base el derecho de los grupos minoritarios a la inclusión (Habermas, 2008a: 270) ${ }^{9}$, su naturaleza moral (Sen, 2010; Cortina, 2009a) permite dejar también un espacio a la argumentación ética (Sen, 2010: 419420) que surge ante la indignación al percibir que son violados. Es esta dimensión moral, que introduce la perspectiva de inclusión igualitaria de todos los seres humanos, la que proporciona fundamento a los derechos humanos (Habermas, 2008a: 54). En el discurso de la CDPD se ahonda en ese fundamento moral, al proclamar que los derechos humanos corresponden también a personas con características corporales o mentales diversas. Por este carácter inclusivo, su reconocimiento está desligado de la posesión de una determinada condición corporal o mental (Degener, 2016). En el preámbulo de la CDPD se introduce la perspectiva inclusiva universal al recordar la igual dignidad inherente y los iguales derechos de "todos los miembros de la familia humana", y al establecer como valores fundamentales el "respeto a la dignidad" de las personas con diversidad funcional y el "respeto por la diferencia y la aceptación de las personas con discapacidad como parte de la diversidad y la condición humanas" (artículo 3, a y d).

En estos principios fundamentales de la CDPD aparece entrelazado el hecho de la diversidad que encierra la condición corporal/mental humana con el valor de la intrínseca dignidad de todos los miembros de la familia humana. Tal hecho no anula, sino que es inseparable del valor de la dignidad de la persona con dicha diversidad: la conjugación de ambos requiere de la actitud moral de respeto hacia la dignidad de la persona en la diversidad que manifiesta. El reconocimiento de la igual dignidad de toda persona -tenga o no algún tipo de impedimento- es el fundamento moral del reconocimiento de iguales derechos humanos (Habermas 2012; Cortina, 2009a) ${ }^{10}$.

También en la Constitución Española (CE) se hace referencia a este fundamento moral cuando se proclama la voluntad de "proteger a todos los españoles en el ejercicio de los derechos humanos" (preámbulo). En el artículo 10.1 se hace referencia a la "dignidad de la persona, los derechos inviolables que le son inherentes" como parte del "fundamento

${ }^{9}$ En el artículo 12.1 de la CDPD se establece que los Estados parte "reafirman que las personas con discapacidad tienen derecho en todas partes al reconocimiento de su personalidad jurídica", lo cual justifica el derecho a la inclusión en la comunidad política.

${ }^{10} \mathrm{~A}$ pesar de los importantes avances en el reconocimiento de los derechos de las personas con discapacidad, como pueda serlo la CDPD, las percepciones negativas profundamente arraigadas sobre el menor valor de la vida de estas personas siguen siendo un obstáculo en todas las sociedades: "Esas percepciones surgen del capacitismo, en cuanto sistema de valores que considera que determinadas características típicas del cuerpo y la mente son fundamentales para vivir una vida que merezca la pena ser vivida" (ONU, 2019c: párr. 9). 
del orden político". Y en el artículo 10.2 se indica que las "normas relativas a los derechos fundamentales y a las libertades que la Constitución reconoce se interpretarán de conformidad con la Declaración Universal de Derechos Humanos y los tratados y acuerdos internacionales sobre las mismas materias ratificados por España”. Por tanto, los derechos fundamentales reconocidos a los ciudadanos han de ser interpretados en conformidad con la CDPD (ratificada en 2008 por España y que, en virtud del artículo 96 de la Constitución, es de obligado cumplimiento en el ordenamiento jurídico interno; igualmente porque fue ratificada por la Unión Europea, a cuya legislación también está sujeta). De lo cual puede colegirse que tales derechos fundamentales se extienden sin ningún tipo de excepción a los ciudadanos con diversidad funcional. Los contenidos expresados en la CE, además de tener una dimensión jurídica, remiten al fundamento moral latente en la DUDH y en la CDPD, mostrando también el carácter moral de los derechos fundamentales reconocidos constitucionalmente. El Comité de Bioética de España recuerda, en su informe sobre las implicaciones éticas para las personas con diversidad funcional en el contexto de la pandemia por la covid19 (2020), que, en situación de extrema escasez de recursos:

\section{[...] ya no se trata de fijar cuál es el mejor criterio científico-médico o cuál es la lex artis de la actuación individual de cada profesional, sino, mucho más allá, de limitar el derecho a la protección de la salud de algunos individuos por la escasez de recursos, la decisión solo puede corresponder única y exclusivamente a la autoridad pública, la única facultada constitucionalmente para limitar y suspender derechos.}

Tal decisión de la autoridad pública está sujeta, como pone de manifiesto dicho comité, también en términos jurídico-políticos, al ordenamiento vigente. Es decir, en último término, a la CE y la CDPD. En tal sentido, el límite jurídico-político de tal decisión, inseparable de su límite moral, lo constituye la prohibición expresa de toda forma de discriminación negativa, incluida la discriminación por discapacidad, según el artículo 2 de la DUDH y el artículo 14 de la CE.

El valor moral y jurídico de la igual dignidad de las personas con diversidad funcional abre la puerta a la lucha contra la discriminación. En el artículo 3 de la CDPD se define la discriminación por discapacidad como:

Cualquier distinción, exclusión o restricción por motivos de discapacidad que tenga el propósito o el efecto de obstaculizar o dejar sin efecto el reconocimiento, goce o ejercicio, en igualdad de condiciones, de todos los derechos humanos y libertades fundamentales en los ámbitos político, económico, social, cultural, civil o de otro tipo.
El rechazo a la discriminación constituye un aspecto nuclear de la CDPD (Degener, 2016). Hay un doble modo de entenderlo: en un sentido pasivo, que consiste en abstenerse de "distinguir, excluir 0 restringir" el igual ejercicio efectivo de los derechos humanos en las personas con impedimentos; y en un sentido activo, requiere de acciones del Estado dirigidas a la potenciación de medidas positivas (por ejemplo, la accesibilidad universal) para transformar la sociedad, creando iguales condiciones para el ejercicio efectivo de los derechos humanos (Degener, 2016). En relación a la salud, el artículo 25 de la CDPD también recoge esta doble dimensión, al indicar que los Estados parte "reconocen que las personas con discapacidad tienen derecho a gozar del más alto nivel posible de salud sin discriminación por motivos de discapacidad. Los Estados parte adoptarán las medidas pertinentes para asegurar el acceso de las personas con discapacidad a servicios de salud" ${ }^{11}$. Este doble sentido impregna el enfoque inclusivo propugnado por la ONU para dar una respuesta inclusiva en el contexto de la covid-19. Dicha respuesta - no únicamente por razones de justicia, también por razones de eficacia sanitariaexige, por un lado, que todas las acciones realizadas "deben prohibir cualquier forma de discriminación basada en la discapacidad" y, por otro, que se sitúen las personas con diversidad funcional en "el centro de la respuesta y que participen como agentes de planificación y ejecución" (ONU, 2020b) ${ }^{12}$.

Desde un punto de vista crítico-normativo, el incumplimiento palmario del derecho a la salud sufrido por las personas con diversidad funcional en dicho contexto encierra, pues, una doble discriminación: no solo se les ha excluido al denegar su igual derecho a la salud; también se les ha discriminado al no haber creado iguales condiciones de acceso para lograr la igual efectividad de este derecho en la crisis vivida ${ }^{13}$. Dicho incumplimiento tiene a la base el prejuicio capacitista y el prejuicio

${ }^{11}$ El informe (ONU, 2018: párr. 72) es igualmente claro al señalar que las personas con discapacidad tienen derecho a gozar del más alto nivel posible de salud sin discriminación. Sin embargo, en la práctica sus condiciones en materia de salud son peores que las de la población general, a causa de factores estructurales, como barreras para acceder a la atención primaria y secundaria, la disponibilidad limitada de servicios y programas específicos para la discapacidad, legislaciones y políticas discriminatorias, los estereotipos negativos, el estigma, la exclusión social y la pobreza.

${ }^{12}$ En el artículo 11 de la CDPD se indica la obligación de los Estados parte de tomar "todas las medidas necesarias para garantizar la seguridad y la protección de las personas con discapacidad en situaciones de riesgo, incluidas situaciones de conflicto armado, emergencias humanitarias y desastres naturales".

${ }^{13}$ Se ha denunciado, en este primer sentido de discriminación, que la "pandemia ha aflorado un cuestionamiento de los derechos humanos, materializado en situaciones de denegación de UCIS o denegación a un traslado a un hospital por razón de edad o de discapacidad o a la generación de tentativas de no asignar estos recursos sobre la base de salvar el sistema sanitario" (CERMI, 2020). También ha quedado de manifiesto este segundo sentido de la discriminación: "Si la información sobre salud pública, el entorno construido, las comunicaciones y tecnologías y los bienes y servicios no son accesibles, las personas con discapacidad no pueden tomar las decisiones necesarias, vivir de forma independiente y aislarse o ponerse en cuarentena de forma segura, ni acceder a los servicios públicos y de salud en igualdad de condiciones con los demás” (ONU, 2020b). 
edadista $^{14}$ (Cortina, 2020), que juzga a las personas con diversidad funcional y a los ancianos como seres cuyas vidas resultan menos útiles, no solo porque se considera que tienen menos que aportar a la sociedad, sino también porque suponen una carga económica para ella ${ }^{15}$. Tales prejuicios, reafirmados en el contexto de las crisis provocadas por la pandemia, tienen su base en el rechazo a la condición humana que se revela en su constitutiva vulnerabilidad (Nussbaum, 2006; Cortina, 2017; 2020) ${ }^{16}$.

La CDPD, documento de promoción de las personas con diversidad funcional como sujetos de iguales derechos humanos (Palacios, 2008; Degener, 2016), abre varias rutas de acción para avanzar en una efectiva inclusión (Sen, 2010; Cortina, 2009b: 228): la ruta legislativa, concretada en la transformación de las normas jurídicas internas de los Estados parte; la ruta ética del reconocimiento, con importante recorrido en la educación y las expresiones culturales, bases para avanzar en una moral inclusiva; y la ruta del activismo, propia de las organizaciones cívicas, ocupadas en la denuncia de las injusticias en el impulso de debates públicos. Tales acciones siguen siendo necesarias, a través de las mencionadas rutas, para una respuesta inclusiva a la covid-19 y a la recuperación posterior (ONU, 2020b).

\subsection{Crítica de la discriminación desde la bioética clínica}

Otra fuente relevante de crítica normativa del mencionado trato injusto es la que proviene del debate existente en la bioética médica. Esta

${ }^{14}$ Existe una clara interseccionalidad entre ambos tipos de prejuicio. El informe (ONU, 2019a: párr. 8) señala que la intersección entre vejez y discapacidad produce formas agravadas de discriminación y violaciones específicas de los derechos humanos de las personas de edad con discapacidad. Las personas de edad con discapacidad están sometidas en mayor medida a la pérdida de poder, la denegación de la autonomía, la marginación y la devaluación. Dado que son percibidas como una "carga" o "menos dignas", a las personas de edad con discapacidad se les concede una prioridad inferior en la política y reciben, si es que los reciben, servicios de menor calidad, especialmente en situaciones de escasez de recursos.

15 Un ejemplo paradigmático son las declaraciones de Dan Patrick, vicegobernador de Texas (Estados Unidos), cuando afirmó, en los comienzos de la crisis pandémica que: "hay abuelos, como él, dispuestos a sacrificar sus vidas para salvar la economía de Estados Unidos" (〈https://www.elmundo.es/internacional/2020/03/24/5e79 d8oafc6c83ea708b4579.html〉).

${ }^{16} \mathrm{El}$ edadismo y el capacitismo comparten raíces y consecuencias comunes, pero la desigualdad en la vejez no es simplemente el resultado de prejuicios capacitistas (ONU, 2019a: párr. 7): el edadismo, considerado como la fijación de estereotipos y prejuicios discriminatorios sobre las personas de edad y la vejez, es una forma específica de opresión que afecta particularmente a las personas de edad, con o sin discapacidad, las cuales suelen ser frecuentemente percibidas como una carga, como dependientes, improductivas, indignas y vulnerables. De acuerdo con el modelo social, la discapacidad se interpreta como una construcción social, pero de manera muy diferente las desigualdades derivadas la vejez son mayoritariamente consideradas como "naturales" o "inevitables". Por lo tanto, las personas de edad con discapacidad se ven discriminadas no solo por su discapacidad, sino también a causa de los estereotipos negativos y estigmatizantes sobre las personas de edad. rama de la bioética se ha entendido desde sus comienzos, en el último tercio del siglo $\mathrm{XX}$, como una ética profesional de principios para guiar la relación médico-paciente en el seno del sistema sanitario, tanto en la investigación como en la asistencia (Gracia, 1989; González, 2016) ${ }^{17}$. Esta lectura restringida del papel de la bioética médica ha sufrido una transformación a comienzos del siglo XXI, cuando se produce un acercamiento de la bioética a los Derechos humanos (Tealdi, 2008). En un camino que comienza con el establecimiento de la Declaración Universal sobre Bioética y Derechos Humanos (Unesco, 2005), la bioética toma como referencia el valor universal de la dignidad del ser humano y su vinculación al reconocimiento y realización de los diferentes tipos de derechos humanos surgidos históricamente.

En virtud de esta ampliación, la bioética se puede concebir como una ética de la vida digna. Es decir, como una ética de las condiciones sociales para una vida que merece ser vivida, de acuerdo con las distintas generaciones de Derechos Humanos (González, 2016). En consecuencia, los principios de la bioética médica han de ser interpretados teniendo como referente último el valor incondicionado de la dignidad humana. Desde esta visión de la bioética, el igual respeto, también en tiempos de pandemia, al derecho humano a la salud de las personas con diversidad funcional y personas ancianas queda fundado en su igual dignidad, y constituye una obligación de justicia que debe ser realizada. Si se entiende dicho derecho humano como una de las condiciones morales de inclusión en la comunidad política (Habermas, 2012), excluir ex ante a dichas personas de la asistencia sanitaria resulta inaceptable porque implica también su exclusión como iguales miembros de la comunidad moral y política (Ministerio de Sanidad, 2020). En la práctica médica enmarcada en el sistema sanitario, esta falta de respeto moral y jurídico-político se traduce en su exclusión como pacientes que merecen ser tratados dignamente. Lo cual implica despreciar e ignorar la información sobre su estado clínico real y sus expectativas objetivas de supervivencia. Siendo este criterio el que debe aplicarse a cualquier persona que necesite ser tratada de la enfermedad pandémica (Auriemma et al., 2020; Cortina, 2020; Ministerio de Sanidad, 2020; Comité de Bioética de España, 2020), entonces esa exclusión clínica irrespetuosa es contraria a los principios de beneficencia y de no maleficencia (González, 2016: 233). Por un lado, se les discrimina en la obtención de los beneficios terapéuticos que pudiera ofrecer el uso de los recursos sanitarios, sean éstos curativos o paliativos, aplicados por personal sanitario especializado (principio de beneficencia). Por otro, se les expone discriminatoriamente al daño que pudiera producir

${ }^{17}$ La pandemia provocada por la covid-19 es, al menos en su origen, una crisis de salud pública que afecta de diferentes formas al conjunto de la población. No obstante, tiene repercusiones clínicas en los individuos. Es en lo que atañe a una práctica clínica excepcional desarrollada en catástrofes (Gracia, 2016), desde la que consideramos aquí la crítica normativa. 
la enfermedad o, al menos, al daño psicológico de una espera incierta y sus consecuencias (principio de no-maleficencia).

También se ha puesto de manifiesto la falta de condiciones de accesibilidad a la información, a las instalaciones, a los servicios y a los programas de respuesta a la covid-19 (ONU, 2020b), lo que entraña una limitación en el ejercicio de una plena e igual libertad en la toma de decisiones de los pacientes para protegerse de la enfermedad (principio de autonomía). Por otra parte, la dignidad del paciente también puede entenderse en el sentido de ser considerado un interlocutor válido, que ejerce su autonomía dialógicamente (Cortina, 1997). Los pacientes, ciudadanos usuarios del sistema sanitario, deben ser escuchados respecto a las normas regulatorias de planificación y asignación de los recursos sanitarios para combatir la pandemia, al estar afectados por ellas ${ }^{18}$. Dichas normas han de ser públicas, sometidas al procedimiento de la argumentación y han de poder ser aceptadas por todos en un consenso racional. El ocultamiento a la opinión pública de la exclusión ex ante de personas con diversidad funcional y personas ancianas, desvelado por la prensa y por las organizaciones representativas de dichas personas ${ }^{19}$ fue otro síntoma de un retroceso desde el modelo social de la discapacidad al modelo de prescindencia (Palacios, 2008; Asís, 2020), no solo en el sentido de que las vidas de las personas con diversidad funcional y de las personas ancianas se consideraron prescindibles, también porque se prescindió de sus voces autónomas.

Por último, se produce una falla clara en el principio de justicia, que atañe al nivel institucional que envuelve la práctica médica. Aunque existen diferentes modos de concebir la justicia sanitaria (Gracia, 1989), hay un cierto consenso en considerar que, en un contexto de pandemia, con gran escasez de recursos sanitarios (incluso de soporte vital), debe aplicarse al menos, aunque no necesariamente como criterio único, el principio de equidad (Ministerio de Sanidad, 2020; Comité de Bioética de España, 2020; Patrão, 2020; Michalowski et al., 2020). Dado el carácter igualitario de la justicia como equidad defendida por Rawls (1979) ${ }^{20}$, la aplicación de

${ }^{18}$ Una de las recomendaciones de la ONU para una respuesta inclusiva a la pandemia indica que deben celebrarse "consultas sustantivas con las personas con discapacidad y las organizaciones que las representan y asegurar su participación activa en todas las fases de la respuesta a la COVID-19 y la recuperación posterior" (ONU, 202ob: 3).

19 El informe CERMI (2020) indica que "se han considerado las consultas y quejas recibidas en el propio CERMI vinculadas a la pandemia, las acciones emprendidas como consecuencia del trabajo genuino de la entidad, las denuncias de personas y entidades colaboradoras, tanto del movimiento asociativo como pertenecientes a distintos ámbitos jurídicos, las noticias publicadas en prensa o difundidas en redes sociales". El principio de publicidad de las normas jurídicas, ya formulado por Kant en La paz perpetua (1985: 61-62), exige transparencia en las normas de priorización de pacientes pues, en caso contrario, no serían validadas bioéticamente (Patrão, 2020; Ministerio de Sanidad, 2020).

${ }^{20} \mathrm{El}$ enfoque de las capacidades desarrollado por A. Sen (2010) y por M. Nussbaum aborda el problema de la justicia con las personas con diversidad funcional, cuestión no tratada por J. Rawls. La teoría este principio implica el rechazo de toda forma de discriminación, al ser esta contraria a la igualdad de oportunidades. Excluir ex ante a ciertos grupos sociales del reparto equitativo de recursos sanitarios resulta contrario al trato equitativo que todo paciente merece en justicia.

Siendo la salud un bien primario que requiere distribución equitativa, la discriminación de las personas con diversidad funcional y personas ancianas en el acceso a la asistencia sanitaria supone un trato irrespetuoso al excluirlas como pacientes con igual dignidad ${ }^{21}$, y produce una quiebra de los principios normativos de la profesión médica. De este modo se acentúa la discriminación social con que ya contaban estos grupos sociales; se exacerban los prejuicios capacitista y edadista ya existentes, lo que contribuye al aumento de la división social; puede agrietarse la confianza de las personas con discapacidad y ancianos en la relación médicopaciente, y se puede causar daño psicológico a los profesionales sanitarios que perciban la injusticia de dicha medida (Auriemma et al., 2020)22. Una bioética fundada en los derechos humanos debe ser también una bioética consciente de la discapacidad (ONU, 2019c). Como tal, constituye una fuente fundamental de crítica normativa de criterios excluyentes que han afectado a las personas con diversidad funcional durante la pandemia ${ }^{23}$. De ahí la importancia de que dichas personas estén representadas tanto en los comités de bioética institucionales como en los comités de ética asistencial de los hospitales, que deben asesorar a los profesionales sanitarios en momentos complicados y con gran incertidumbre (Comité de Bioética de España, 2020; Ministerio de Sanidad, 2020), de forma que sus argumentos puedan ser escuchados (ONU, 2019C).

parcial de la justicia social de Nussbaum extiende igualitariamente a las personas con diversidad funcional los mínimos de justicia en las capacidades u oportunidades reales de salud y mantenimiento de la vida (Nussbaum, 2007).

${ }^{21}$ El capacitismo y el edadismo están profundamente arraigados en el pensamiento social, en las políticas, las leyes, las instituciones, las actitudes y las creencias (ONU, 2018: párr. 38; 2019a: párr. 19). Las afecciones y los síntomas médicos de las personas con discapacidad suelen ser vistas como parte de su deficiencia, lo que a menudo conlleva la falta de tratamiento, o la estimación de que estas personas no merecen tratamiento. Por otra parte, en las personas de edad sus deficiencias se consideran como una consecuencia natural del envejecimiento, por lo que las barreras que les afectan en los ámbitos de su participación social no se perciben como una construcción social susceptible de eliminarse, sino como un hecho natural de su condición actual de vida.

${ }^{22}$ Hasta ahora gran parte de la labor realizada en el campo de la bioética se ha basado en un entendimiento insuficiente e inexacto de la diversidad y la complejidad social de la discapacidad, y los trabajos bioéticos sobre la discapacidad se redactan en su mayoría desde el punto de vista de las personas sin discapacidad (ONU, 2019c: párr. 20). Las creencias capacitistas, como que las personas con discapacidad tienen menos valor que las demás, o que sus vidas no merecen la pena ser vividas, protagonizan la mayoría de las deliberaciones bioéticas, de manera que no tienen en cuenta las cuestiones bioéticas que realmente preocupan a las personas con discapacidad.

${ }^{23}$ Tanto el informe bioético del Ministerio de Sanidad (2020) como el informe del Comité de Bioética de España (2020) se han pronunciado críticamente sobre la discriminación de las personas con diversidad funcional y de las personas ancianas, apelando a un planteamiento de universalismo moral. 


\section{Una respuesta inclusiva a la pandemia}

Una bioética de derechos humanos que cuestione el capacitismo y el edadismo en la respuesta a la covid-19 ha de considerar, en un sentido críticopositivo, dos cuestiones relevantes para la protección normativa de la dignidad personal: la cuestión de las normas ex post de priorización de pacientes en contextos de catástrofe sanitaria y la cuestión de los determinantes sociales de salud como prevención de la salud pública. Aunque el foco de la mayoría de las reflexiones bioéticas se ha centrado en la primera cuestión, también es necesario tener presente la segunda.

\subsection{Priorización sanitaria y dignidad humana}

La priorización de pacientes, o triaje, forma parte de la práctica clínica ordinaria. Ahora bien, en una situación de catástrofe sanitaria, el triaje no se reduce a llevar a cabo una mera clasificación de pacientes, como ocurre en aquella. En una situación excepcional, cuando la catástrofe sanitaria conlleva escasez extrema de recursos y, como consecuencia, se han de tomar decisiones trágicas, el triaje consiste en la selección de pacientes a los que aplicar los recursos limitados existentes (Gracia, 2016: 80) ${ }^{24}$. Desde nuestro punto de vista, las normas que se establezcan para esa situación excepcional deberán evitar discriminaciones negativas y cargas clínicas desproporcionadas sobre categorías de personas, consiguiendo una protección inclusiva de la dignidad humana.

El análisis de la priorización, a partir de la reciente literatura sobre el tema, lleva a considerar la imbricación de cuestiones epistémicas y normativas. Una primera cuestión que se plantea tiene que ver con su ámbito de origen. Aunque su establecimiento corresponda al terreno político-administrativo, teniendo en cuenta orientaciones procedentes del ámbito tecno-científico de salud pública, resulta inadecuado adoptar un punto de vista positivista. Dichas normas no deberían establecerse remitiéndose en exclusiva a puros hechos a los que haya que atender en la gestión sanitaria de la pandemia (recursos disponibles, coste económico, aplicación ante mayor vulnerabilidad física, etc.). No se trata de meras normas pragmáticas (Habermas, 2000: 111), que únicamente hagan referencia a los medios escasos disponibles para alcanzar la finalidad de un uso eficaz de ellos. En la medida en que esta eficacia está intrínsecamente conectada a salvar vidas humanas amenazadas por la pandemia, responden a un conflicto de intereses que se resume en la pregunta: ¿qué vidas deben salvarse? (Michalowski et al., 2020). Por tanto, al afectar a vidas humanas y repercutir en el derecho

${ }^{24}$ Tal es el caso, por ejemplo, de la limitación de acceso a las UCI o del uso racionado de respiradores en pacientes con una covid-19 agravada, lo que obligó a seleccionar pacientes a los que aplicar estos recursos de soporte vital. a la salud pública, no están desligadas de valores morales, ya que los hechos que deben ser atendidos se entremezclan con valores como la justicia en el ámbito de instituciones sanitarias (Conill, 2005), remitiendo, en último término, a la igual dignidad de cada uno de los pacientes.

Las normas de asignación de recursos sanitarios no son, pues, normas puramente científico-técnicas, sino que, aun dependiendo del conocimiento de los hechos, son normas morales (Patrão, 2020). Implican una elección que ha de resultar correcta armonizando la salud de la población con la igual dignidad y el igual derecho a la salud de los individuos. Han de ser justas en un reparto equitativo de beneficios y cargas clínicos compatible con el igual interés de todos en conservar la salud. En consecuencia, aunque la decisión sobre las normas justas de asignación de recursos corresponda a los responsables políticoadministrativos, es insuficiente contar con el conocimiento de los expertos científicos. Todos los usuarios del sistema sanitario, incluyendo a las personas con diversidad funcional y las personas ancianas, en tanto que afectados por ellas, han de poder realizar sus aportaciones en el previo proceso deliberativo ${ }^{25}$. Su validez dependerá, de acuerdo con la ética del discurso, de que todos los usuarios del sistema sanitario, en tanto que interlocutores válidos, pudieran dar su consentimiento (Habermas, 2000: 36; Cortina, 1997: 236-240).

El carácter problemático de la aplicación de las normas que regulan dicha situación radica en la imposibilidad fáctica de salvar la vida de todos los pacientes. En tal circunstancia, el deber de intentar salvar la vida de todos los pacientes graves no puede ser realizado. En esta excepcional circunstancia se requiere que el enfoque deontológico sea combinado con un enfoque utilitarista: existe el deber de salvar el mayor número posible de vidas humanas, aunque bajo la condición de respetar la igual dignidad de los pacientes (Auriemma et al., 2020; Bagenstos, 2020; Solomon et al., 2020; Patrão, 2020; Michalowski et al., 2020; Mello, Govind y White, 2020). La justicia de las normas de exclusión de casos individuales en el citado contexto, con el objetivo de maximizar las vidas de la población, estriba en que se pudiera producir una justificación y aplicación imparcial de estas, de modo que se atendiera a los intereses de todos en la misma medida (Habermas, 2011: 292). En este caso, la condición de imparcialidad radica, además de en el reparto equitativo de los beneficios y las cargas clínicas, en que todos los pacientes tengan una atención singularizada, considerando únicamente su estado clínico y sus expectativas objetivas de vida, basadas en la evidencia (Ministerio de Sanidad, 2020; Romeo y Urruela, 2020). El igual

${ }^{25}$ En tal sentido, Patrão (2020) afirma que "la justicia social [en la asignación de recursos] debe ser vista como un proceso deliberativo, plural, inclusivo y permanentemente abierto". Por su parte, Romeo y Urruela (2020) consideran que, idealmente, dichas normas deberían ser diseñadas "de antemano al advenimiento de una pandemia permitiendo la participación abierta y el debate experto en el diseño de las pautas de priorización". 
respeto a la dignidad de los pacientes tendrá que mantenerse, exclusivamente en función del resultado de dicho examen clínico, con la decisión posterior sobre el uso de medios de soporte vital o, cuando esto no sea posible y el paciente no sea considerado prioritario, con el uso de medios paliativos de acompañamiento (Romeo y Urruela, 2020).

El establecimiento de normas de priorización de pacientes y la decisión médica de su aplicación no debería recurrir al uso de características personales (edad, impedimento, etc.) no directamente relacionadas con la situación clínica de los pacientes, si bien algunos expertos consideran que pueden usarse como criterios secundarios complementarios en caso de desempate (Auriemma et al., 2020; Solomon et al., 2020, Patrão, 2020). Además de que puedan resultar irrelevantes respecto a los conocimientos derivados del examen clínico, resulta inaceptable su uso normativo prima facie, puesto que la apelación a la igual dignidad personal resulta independiente de toda característica fáctica individual. En este sentido, Patrão (2020) contrapone dos modos diferentes de entender la priorización de los medios sanitarios: el "racionamiento", que limita los medios en función de las características personales, y la "racionalización" u optimización de los recursos sanitarios para maximizar los beneficios que obtendrán los pacientes a partir de su situación clínica, sin considerar sus características personales. Solo este segundo proceder hace compatible el enfoque utilitarista con el igual respeto a la dignidad de los pacientes.

Por último, en la valoración clínica de los pacientes son importantes algunas cuestiones epistémicas referidas al cálculo en la maximización de los beneficios clínicos. Se ha considerado que resulta más aceptable el uso de la probabilidad de supervivencia a corto plazo o de la probabilidad inmediata de sobrevivir a la enfermedad, frente a la aplicación de años de vida o de años de vida de calidad (Auriemma, 2020; Solomonn et al., 2020; Mello,Govind y White, 2020). El uso de dicho criterio resulta más coherente con una atención clínica singularizada abstraída de consideraciones externas, evitando así que en los juicios clínicos se deslice subrepticiamente el prejuicio capacitista o edadista. El uso de criterios pretendidamente objetivos para la asignación de recursos puede estar contaminado con sesgos subjetivos acerca de la menor calidad de vida de las personas con diversidad funcional (Auriemma et al., 2020; Bagenstos, 2020). Además del carácter erróneo de este sesgo subjetivo, que puede estar presente en orientaciones bioéticas o en la economía de la salud (Amundson, 2010), no tiene en cuenta que lo "que determina la calidad de vida de las personas con discapacidad no es la deficiencia en sí, sino las mismas cosas que determinan la calidad de vida de las personas sin discapacidad" (ONU, 2019c). Es decir, no tiene en cuenta cómo la vida de las personas con deficiencia puede resultar adecuadamente acogida por los arreglos sociales (Sen, 2002a: 58).
En una atención clínica singularizada, que considere exclusivamente las posibilidades de supervivencia a la enfermedad a corto plazo, la base cognitiva del juicio médico viene a concordar, por medio de la obligación deontológica a que se sujeta dicho juicio ${ }^{26}$, con la apreciación normativa del respeto a la igual dignidad de cada paciente. La racionalización de recursos (Patrão, 2020) en provecho de quienes, en tal situación de extrema escasez, podrán beneficiarse más de los tratamientos frente a la pandemia, hace compatible la búsqueda de la maximización de vidas de la población con el respeto a la igual dignidad de cada persona. Teniendo en cuenta lo anterior, en una situación extraordinaria de pandemia, puede resultar moralmente aceptable la exclusión ex post de una persona con diversidad funcional o una persona anciana. Lo será únicamente cuando no pueda beneficiarse del tratamiento, bien porque su impedimento interfiera con el tratamiento o porque, aunque éste pueda tener éxito, su impedimento o su comorbilidad pueda producir su muerte en un plazo cercano (Bagentos, 2020).

\subsection{Determinantes sociales de salud}

Una visión bioética centrada en los derechos humanos que adopte un punto de vista prospectivo e inclusivo de respuesta a la pandemia no debe focalizarse en exclusiva en la cuestión de la priorización de los pacientes. Yendo más allá de la vertiente clínica, este enfoque panorámico ha de incidir en la conexión entre la salud de la población y los factores institucionales y de práctica social que repercuten en ella.

Aunque es un hecho que la suma de múltiples factores que afectan a las personas con diversidad funcional y ancianas (comorbilidad, condición de dependencia, mayores necesidades asistenciales, etc.) ha aumentado el riesgo de afectación de la enfermedad pandémica, no se trata de un hecho inmutable. En él se produce una interacción, sin discontinuidad, entre causas naturales y decisiones sociales previas. La existencia de esta doble causalidad entremezclada conlleva que, aunque la pandemia pueda interpretarse como vulnerabilidad natural, detrás de su desigual distribución aflora una injusta vulnerabilidad social, que requiere corregirse, tanto por razones de justicia como por la eficacia de las medidas de control de la pandemia. En este sentido, la epidemiología social pone al descubierto los denominados determinantes sociales de la salud ${ }^{27}$, cuestión bioética que trasciende

\footnotetext{
26 “El carácter deontológico del juicio, que orienta la práctica médica, está confirmado por la obligación de todos los miembros del cuerpo médico, en general, de proporcionar ayuda no solo a sus pacientes, sino a toda persona enferma o herida que se encuentre en situación de peligro" (Ricoeur, 2008). En una situación sanitaria excepcional, las limitaciones de curación debida a la insuficiencia de los recursos sanitarios no anulan la obligación del cuidado médico, sino que la redirigen, en función de los juicios clínicos singularizados y de los recursos disponibles, hacia la maximización de vidas de la población.

${ }^{27}$ La OMS publicó en 2008 el famoso Informe de la Comisión sobre determinantes sociales de la salud, liderado por M. Marmot.
} 
la perspectiva clínica para abrirse al ámbito de la justicia social (Puyol, 2016). Dada la conexión entre la salud y la justicia social (Sen, 2002b), las injustas condiciones sociales previas son las que han contribuido a una afectación desproporcionada de la covid-19 en las personas con diversidad funcional y ancianas.

El enfoque de las capacidades de Sen y Nussbaum constituye una concepción de la justicia que subraya la importancia de los arreglos sociales en la calidad de vida de las personas con diversidad funcional (Sen, 2010; Nussbaum, 2007). Para este enfoque, la justicia se concibe en términos de capacidades $u$ oportunidades reales para elaborar libremente planes de vida que una persona tiene razones para valorar. En las capacidades confluyen tanto las características personales como las aportaciones de las instituciones sociales. Sen pone un ejemplo epidemiológico oportuno al tema que nos ocupa: la capacidad o libertad de disfrutar una vida libre de paludismo. En este ejemplo muestra Sen que la libertad de vivir como una persona quiere no depende exclusivamente de la elección personal, sino que "es fortalecida por la política pública que transforma a los ambientes epidemiológicos y social” (Sen, 2002a: 72). Las políticas públicas que contribuyan a aportar un trato más justo con los ancianos y las personas con diversidad funcional generarán mejores condiciones sociales para ellos que, a su vez, reforzarán la salud pública de dichos grupos sociales. Dichas políticas públicas habrán de incidir en aquellas condiciones sociales que han agravado la salud pública de estos grupos sociales en la pandemia. Sin ánimo de exhaustividad, apuntamos los siguientes aspectos:

a. Uno de los factores que han contribuido a la propagación de la pandemia en los grupos de personas con diversidad funcional y personas ancianas ha sido la institucionalización en residencias. Por un lado, la concentración de personas en este tipo de entorno físico se ha traducido en un factor de riesgo para la transmisión de la enfermedad. Por otra parte, las insuficiencias estructurales de las residencias respecto a un buen cuidado saludable han actuado como multiplicadores de este riesgo (Médicos sin fronteras, 2020; CERMI, 2020; Guidry-Grimes et al., 2020), lo que ha conllevado que un alto porcentaje de la mortalidad total se concentre en miembros institucionalizados de estos grupos sociales. Las políticas públicas no solo han de incidir en la transformación estructural de las residencias para prevenir pandemias, sino que también han de favorecer la vida independiente en iguales condiciones, de acuerdo con el artículo 19 de la CDPD. En este sentido, Nussbaum relaciona la capacidad de salud física con el disponer de un lugar adecuado para vivir (Nussbaum, 2007: 88), lo que exige contar con alternativas que permitan sortear las preferencias adaptativas, es decir, que las personas renuncien a otras alternativas mejores y se queden con aquellas únicas que se les ofrecen (Nussbaum, 2007: 193).

b. Las insuficiencias en el desarrollo del derecho a la accesibilidad universal (artículo 9 de la CDPD) también han sido destacadas como factor social (CERMI, 2020; Guidry-Grimes et al., 2020) que ha influido negativamente en la prevención de la enfermedad en estos grupos sociales. Aquí se incluyen no solo las características de los entornos físicos, de los medios de comunicación o de los mensajes emitidos, sino también las personas encargadas de la asistencia. La concepción integral de la asistencia defendida por Nussbaum (2007) incluye la asistencia en las diferentes capacidades. La capacidad de vivir y de contar con buena salud requiere disponer, cuando resulte imprescindible, de asistencia adecuada y formada para poder protegerse de la pandemia. Previamente, el diseño de entornos físicos, de comunicación o cognitivos accesibles contribuye a favorecer en muchos casos la autonomía en la adopción de medidas de autoprotección.

c. La pobreza constituye un factor social que condiciona la salud general en diferentes grupos sociales. El mayor diferencial de desempleo y de pobreza que tienen las personas con diversidad funcional respecto al resto de ciudadanos, además de incidir en una mayor comorbilidad, conlleva falta de recursos destinados a medidas higiénicas frente a la pandemia. El artículo 28 de la CDPD compromete a los Estados parte a realizar políticas de reducción de la pobreza para facilitar un nivel de vida adecuado.

d. La subrepresentación política de las personas con diversidad funcional en la toma de decisiones que les afectan para el freno de la expansión de la pandemia se traduce en que sus intereses pueden quedar marginados. El artículo 4 de la CDPD obliga a los Estados parte a que en todas las políticas desarrolladas se tengan en cuenta los derechos de las personas con diversidad funcional. Las políticas de salud pública no son una excepción. Por otra parte, en el preámbulo, apartado o, se considera que deben participar activamente en las decisiones sobre políticas que les afecten directamente. El ejercicio de esta autonomía pública (directamente, con asistencia o mediante representación) resulta crucial para una salida inclusiva de la pandemia (ONU, 2020b).

\section{Epílogo}

Las dramáticas circunstancias de salud pública vividas durante la primera etapa de la pandemia de la covid-19 encerraron en su seno, como se ha puesto de manifiesto desde organismos internacionales y organizaciones civiles, una no menos dramática crisis de civilización. En el seno de sociedades con fuerte dosis de individualismo, afloró con crudeza el prejuicio capacitista y el prejuicio edadista. El universalismo moral y político que toma como 
fundamento la igual dignidad de toda persona y que exige el reconocimiento de sus iguales derechos confronta el trato recibido por personas ancianas y con diversidad funcional con la perspectiva de la justicia, tanto en el ámbito sanitario como en el ámbito social. La fuerza moral subyacente en la positivización de los derechos de la DUDH y de la CDPD, que impregna también una reflexión bioética centrada en ellos, genera rutas de reivindicación de una plena inclusión social de dichas personas en la respuesta a la pandemia. La normatividad bioética orientadora de la práctica clínica exige, a la luz del universalismo, la complementación del enfoque maximizador de la salud de la población con el deontologismo igualitario que rechaza la exclusión o relegación de personas de grupos sociales estigmatizados. Por su parte, la existencia contrastada de bases sociales de la salud apunta a la necesidad de políticas sociales justas que reestructuren las condiciones sociales de vida de las personas que forman parte de estos grupos sociales, como modo de proteger integralmente su salud.

Únicamente el reconocimiento efectivo de los derechos humanos podrá posibilitar la reducción del capacitismo y el edadismo. A su vez, solo la extensión de la conciencia de la igual dignidad en la diferencia impulsará una sociedad estructuralmente más justa. Ambas condiciones constituyen requisitos para una respuesta inclusiva a la pandemia. 
AMUNDSON, R. (2010): "Quality of life, disability, and hedonic psychology", Journal for the Theory of Social Behaviour, pp. 374-392.

ARBOLEDA, J. (2000): "Equidad y asignación de recursos: donde la ética y la justicia social se interceptan”, en Lolas, F. (edit.), Bioética y cuidado de la salud. Equidad, Calidad, Derechos, Santiago de Chile, Programa Regional de Bioética OPS/OMS, pp. 53-67.

ASís, R. (2020): “La prescindencia está aquî”, «https:// pasocer0243055203.com/>.

AURIEMMA, C. et al. (2020): "Eliminating Categorical Exclusion Criteria in Crisis Standards of Care Frameworks", The American Journal of Bioethics, 20: 7, pp.28-36.

BAGENSTOS, S. (2020): May hospitals withhold ventilators from covid-19 patients with pre-existing disabilities? Notes on the law and ethics of disability-based medical rationing, Yale Law Journal Forum (2020, forthcoming).

CAMPBELL, F.K. (2001): “Inciting Legal Fictions: Disability’s Date with Ontology and the Ableist Body of the Law", Griffith Law Review, 10, pp. 42-62.

- (2008): "Refusing Able(ness): A Preliminary Conversation about Ableism", M/C Journal, 11(3).

CERMI (2020): El impacto de la pandemia del coronavirus en los derechos humanos de la personas con discapacidad en España, Madrid, Cinca.

CHERNEY, J.L. (2011): “The Rhetoric of Ableism”, Disability Studies Quarterly, 31(3).

COMITÉ DE BIOÉTICA DE ESPAÑA (2020): Informe del Comité de Bioética de España sobre los aspectos bioéticos de la priorización de recursos sanitarios en el contexto de la crisis del coronavirus, 〈http://assets.comitedebioetica. es/files/documentacion/Informe\%2oCBE-\%20 Priorizacion\%2ode\%2orecursos\%2osanitarioscoronavirus\%20CBE.pdf〉.

CONILL, J. (2005): “Justicia sanitaria”, en GÓMEZ-HERAS, J.M. y VELAYOS, C. (edit.), Bioética. Perspectivas emergentes y nuevos problemas, Madrid, Tecnos.

CORTINA, A. (2009a): Las fronteras de la persona, Madrid, Taurus.

- (2009b): Ética de la razón cordial. Educar en la ciudadanía en el siglo XXI, Oviedo, Ediciones Nobel.

- (2017): Aporofobia, el rechazo al pobre. Un desafío para la democracia, Barcelona, Paidós.

- (2020): “Desenmascarar la Gerontofobia”, El país, 22-72020.

DEGENER, T. (2016): “Disability in a Human Rights Context”, Laws, 5, 35, <doi: https://doi.org/10.3390/ laws5030035’.

GONZÁLEZ, G. (2016): Bioética: un nuevo paradigma. De la ética aplicada a la ética de la vida digna, Madrid, Tecnos.

GRACIA, D. (1989): Fundamentos de bioética, Madrid, Eudema.

- (2016): "La ética en las situaciones trágicas: urgencias, emergencias y catástrofes”, Eidon, 46, pp. 70-82, <doi: https://doi.org/10.13184/ eidon.46.2016.70-82〉.

GUIDRY-GRIMES, L. et al. (2020): “Disability Rights as a Necessary Framework for Crisis Standards of Care and the Future of Health Care,", Hastings Center Report, 50, 3: pp. 28-32.

HABERMAS, J. (2000): Aclaraciones a la ética del discurso, Madrid, Trotta. 
- (2008a): Entre naturalismo y religión, Barcelona, Paidós.

- (2008b): La inclusión del otro, Barcelona, Paidós.

- (2011): Verdad y justificación, Madrid, Trotta.

- (2012): “El concepto de dignidad humana y la utopía realista de los derechos humanos", en $L a$ constitución de Europa, Madrid, Trotta.

KANT, I. (1985): La paz perpetua, Madrid, Tecnos.

LINTON, S. (1998): Claiming Disability: Knowledge and Identity, New York, New York University Press.

MCRUER, R. (2016): “Lo Queer y lo Crip, como formas de re-apropiación de la dignidad disidente. Una conversación con Robert McRuer", Dilemata. Revista Internacional de Éticas Aplicadas, 20, pp. 137-144.

MÉDICOS SIN FRONTERAS (2020): Poco, tarde y mal. El inaceptable desamparo de los mayores en las residencias durante la COVID-19 en España, 〈http://static.msf.es/web/archivos/cov-19/ AAFF-MSF-Informe-COVID19-Residencias-BAJA. pdf?_ga=2.140416691.1834597249.15976545182136340315.1553527222\& $g a c=1.79696997 .1597214225 . j o K C Q j w g 8 n_{5} B R C$ dARIsALxKb96ox39jqD7V1IZUZKIFEZSOVT5 vRkYbvwAw3RsZ7-ZNkbQUVeFwJsaAinzEALw_ $w C B>$.

MELLO, M.; GOVIND J.D. y WHITE D. (2020): “Respecting Disability Rights Toward Improved Crisis Standards of Care", The New England Journal of Medicine, <https://doi.org/10.1056/ NEJMp2011997>.

MICHALOWSKI, S. et al. (2020): “Triage in the COVID-19 Pandemic: Bioethical and Human Rights Considerations", Joint Technical Report of the Essex Autonomy Project and the Ethics of Powerlessness Project, <http://repository. essex.ac.uk/27292>.

MINISTERIO DE SANIDAD (2020): Informe sobre los aspectos éticos en situaciones de pandemia: el SARS-CoV-2, 2. 〈https://www.mscbs. gob.es/profesionales/saludPublica/ ccayes/alertasActual/nCov-China/ documentos/200403-INFORME_PANDEMIAFINAL-MAQUETADO.pdf〉.

NUSSBAUM, M. (2007): Las fronteras de la justicia, Barcelona, Paidós.

ONU (2018): Informe de la Relatora Especial sobre los derechos de las personas con discapacidad (A/73/161). El derecho a la salud, <https:// undocs.org/es/A/73/161).

- (2019a): Informe de la Relatora Especial sobre los derechos de las personas con discapacidad $(A / 74 / 186)$. La intersección entre envejecimiento y discapacidad, 〈https:// undocs.org/es/A/74/186>.

- (2019b): La toma de conciencia a tenor del artículo 8 de la Convención sobre los Derechos de las Personas con Discapacidad, Informe de la Oficina del Alto Comisionado de las Naciones Unidas para los Derechos Humanos (A/HRC/43/27), 〈https:// undocs.org/es/A/HRC/43/27>.

- (2019c): Informe de la Relatora Especial sobre los derechos de las personas con discapacidad (A) $H R C / 43 / 41)$. Los efectos del capacitismo en la práctica médica y científica, 〈https://undocs. org/es/A/HRC/43/41>.

- (2020a): Shared responsibility, global solidarity: Responding to the socio-economic impacts of COVID-19, Nueva York <https://unsdg.un.org/ sites/default/files/2020-03/SG-Report-SocioEconomic-Impact-of-Covid19.pdf〉.

- (2020b): Policy Brief: A Disability-Inclusive Response to COVID-19, Nueva York,

〈https://unsdg.un.org/sites/default/ files/2020-05/Policy-Brief-A-DisabilityInclusive-Response-to-COVID-19.pdf〉.

- (2020c): Covid-19 y los derechos de las personas con discapacidad: directrices, <https://www.ohchr. org/Documents/Issues/Disability/COVID-19 and_The_Rights_of_Persons_with_Disabilities_ SP.pdf〉.

- (2020d): “COVID-19: Bachelet insta a los Estados a que adopten medidas adicionales para incluir a las personas con discapacidad", «https:// www.ohchr.org/SP/NewsEvents/Pages/ DisplayNews.aspx?News|D=25847\&Lang|D=S〉.

PARLAMENTO EUROPEO (2020): Resolución de 18 de junio de 2020, sobre la Estrategia Europea sobre Discapacidad posterior a 2020 (2019/2975(RSP)), 〈https://www.europarl. europa.eu/doceo/document/TA-9-2020-0156_ ES.html>.

PATRÃO, M.C. (2020): "Ethical health resources allocation: Why the distinction between 'rationing' and 'rationalization' matters”, Revista de Bioética y Derecho, 50, pp. 63-79.

PUYOL, Á. (2016): “Ética y epidemiología social”, en PUYOL, À. y ESTANY, A. (Ed.) (2016): Filosofía de la epidemiología social, Madrid, Plaza y Valdés.

RAWLS, J. (1979): Teoría de la justicia, Madrid, F.C.E.

RICOEUR, P. (2008): Lo justo 2, Madrid, Trotta.

ROMEO, C.M. y URRUELA, A. (2020): "La gestión de soportes vitales escasos en contextos de pandemia. Reflexiones acerca de la aplicación de triajes en el marco de la COVID-19", Revista de Bioética y Derecho, 50, pp. 99-112.

SEN, A. (2002a): "Capacidad y bienestar", en NUSSBAUM, M. y SEN, A, (comp.): La calidad de vida, México D.F., FCE.

- (2002b): “Why health equity?”, Health Economics, 11, pp. 659-666.

- (2010): La idea de la justicia, Madrid, Taurus.

SOLOMON, M. et al. (2020): “Covid-19 Crisis Triage Optimizing Health Outcomes and Disability Rights", The New England Journal of Medicine, 2020, 〈https:doi.org/10.1056/NEJMp2008300〉.

SUÁREZ, A.; MATÍAS, J. y COMAS, A. (2020): Impact and mortality of the first wave of COVID-19 on people living with dementia in Spain, International Long Term Care Policy Network.

TEALDI, J.C. (2010): "Bioética de los Derechos Humanos" (voz), en TEALDI, J. C. (dir.): Diccionario latinoamericano de bioética, Bogotá, UNESCO/ Universidad Nacional de Colombia.

UNESCO (2005): Declaración Universal sobre Bioética y Derechos humanos, 〈http://portal.unesco. 
org/es/ev.php-URL_ID=31058\&URL_DO=DO TOPIC\&URL_SECTION=201.html.

WOLBRING, G. (2008a): "Is There an End to Out-Able? Is There an End to the Rat Race for Abilities?", $M / C$ Journal, 11(3).
- (2008b): “The Politics of Ableism”, Development, 51, pp. 252-258.

WOLBRING, G. y GUZMÁN, P. (2010): "Human Enhacement Through the Ableism Lens (an e-mail interview made by Francisco Guzmán)", Dilemata. Revista Internacional de Éticas Aplicadas, 3, pp. 1-13. 